\title{
A Note on Hermite poly-Bernoulli Numbers and Polynomials of the Second Kind
}

\author{
Waseem A. Khan ${ }^{1, *}$, N. U. Khan², Sarvat Zia ${ }^{1}$ \\ ${ }^{1}$ Department of Mathematics, Integral University, Lucknow-226026, India \\ ${ }^{2}$ Department of Applied Mathematics, Faculty of Engineering and Technology, Aligarh Muslim University, Aligarh, India \\ *Corresponding author: waseem08_khan@rediffmail.com
}

Received August 06, 2015; Revised September 24, 2015; Accepted October 02, 2015

\begin{abstract}
In the paper, we introduce a new concept of poly-Bernoulli numbers and polynomials of the second kind which is called Hermite poly-Bernoulli numbers and polynomials of the second kind. We also investigate and analyse its applications in number theory, combinatorics and other fields of mathematics. The results derived here are a generalization of some known summation formulae earlier studied by Jolany et al. [17,18], Dattoli et al [14] and Pathan et al [29,30].
\end{abstract}

Keywords: Hermite polynomials, poly-Bernoulli polynomials of the second kind, Hermite poly-Bernoulli polynomials of the second kind, summation formulae, symmetric identities

Cite This Article: Waseem A. Khan, N. U. Khan, and Sarvat Zia, "A Note on Hermite poly-Bernoulli Numbers and Polynomials of the Second Kind." Turkish Journal of Analysis and Number Theory, vol. 3, no. 5 (2015): 120-125. doi: 10.12691/tjant-3-5-2.

\section{Introduction}

The 2-variable Kampe de Feriet generalization of the Hermite polynomials [13] and [14] reads

$$
H_{n}(x, y)=n ! \sum_{r=0}^{\left[\frac{n}{2}\right]} \frac{y^{r} x^{n-2 r}}{r !(n-2 r) !}
$$

These polynomials are usually defined by the generating function

$$
e^{x t+y t^{2}}=\sum_{n=0}^{\infty} H_{n}(x, y) \frac{t^{n}}{n !}
$$

and reduce to the ordinary Hermite polynomials $H_{n}(x)$ (see [1]) when $y=-1$ and $x$ is replaced by $2 x$.

The classical Bernoulli numbers $B_{n}$. Bernoulli polynomials $B_{n}(x)$ and their generalization $B_{n}^{(\alpha)}(x)$ (of real or complex) of order $\alpha$ are usually defined by means of the following generating functions ([2,40,41,42]).

$$
\begin{gathered}
\left(\frac{t}{e^{t}-1}\right)=\sum_{n=0}^{\infty} B_{n} \frac{t^{n}}{n !} \\
\left(\frac{t}{e^{t}-1}\right) e^{x t}=\sum_{n=0}^{\infty} B_{n}(x) \frac{t^{n}}{n !}
\end{gathered}
$$

and

$$
\left(\frac{t}{e^{t}-1}\right)^{\alpha} e^{x t}=\sum_{n=0}^{\infty} B_{n}^{(\alpha)}(x) \frac{t^{n}}{n !}\left(|t|<2 \pi ; 1^{\alpha}=1\right) .
$$

The $B_{n}$ are rational numbers and in particular $B_{n}^{(1)}(0)=B_{n}(0)=B_{n}$.

As is well known, the Bernoulli polynomials of the second kind [35] are defined by the generating function to be

$$
\frac{t}{\log (1+t)}(1+t)^{x}=\sum_{n=0}^{\infty} b_{n}(x) \frac{t^{n}}{n !} .
$$

When $x=0, b_{n}=b_{n}(0)$ are called the Bernoulli numbers of the second kind. The first few Bernoulli numbers bn of the second kind are $b_{0}=1, b_{1}=1 / 2, b_{3}=1 / 24, b_{4}=-19 / 720$, $b_{5}=3 / 160, \ldots$.

In [22], Kaneko introduced and studied poly-Bernoulli numbers which generalize the classical Bernoulli numbers. poly-Bernoulli numbers $B_{n}^{(k)}$ with $k \in z$ and $n \in N$, appear in the following power series

$$
\frac{L i_{k}\left(1-e^{-t}\right)}{1-e^{-t}}=\sum_{n=0}^{\infty} B_{n}^{(k)} \frac{t^{n}}{n !}
$$

where $k \in Z$ and

$$
L i_{k}=\sum_{m=1}^{\infty} \frac{z^{m}}{m^{k}},|z|<1
$$

so for $k \leq 1$,

$$
L i_{k}=-\ln (1-z), L i_{0}(z)=\frac{z}{1-z}, L i_{-1}=\frac{z}{(1-z)^{2}}, \ldots
$$

Moreover when $k \geq 1$, the left hand side of (1.1) can be written in the form 
$e^{t} \frac{1}{e^{t}-1} \int_{0}^{t} \frac{1}{e^{t}-1} \cdots \int_{0}^{t} \frac{1}{e^{t}-1} \int_{0}^{t} \frac{t}{e^{t}-1} d t d t \cdots d t=\sum_{n=0}^{\infty} B_{n}^{(k)} \frac{t^{n}}{n !}$

In the special case, one can see

$$
B_{n}^{(1)}=B_{n} .
$$

Recently, Jolany et al $[17,18]$ generalized the concept of poly-Bernoulli polynomials is defined as follows.

Let $a, b, c>0$ and $a \neq b$. The generalized polyBernoulli numbers $B_{n}^{(k)}(a, b)$, the generalized polyBernoulli polynomials $B_{n}^{(k)}(x, a, b)$ and the polynomials $B_{n}^{(k)}(x, a, b, c)$ are appeared in the following series respectively

$$
\begin{aligned}
& \frac{L i_{k}\left(1-(a b)^{-t}\right)}{b^{t}-a^{-t}}=\sum_{n=0}^{\infty} B_{n}^{(k)}(a, b) \frac{t^{n}}{n !}|t|<\frac{2 \pi}{|\ln a+\ln b|}(1.8) \\
& \frac{L i_{k}\left(1-(a b)^{-t}\right)}{b^{t}-a^{-t}} e^{x t}=\sum_{n=0}^{\infty} B_{n}^{(k)}(x, a, b) \frac{t^{n}}{n !}, \quad(1.9) \\
& \frac{|t|<\frac{2 \pi}{|\ln a+\ln b|}}{L_{k}\left(1-(a b)^{-t}\right)} \\
& b^{t}-a^{-t} \\
& c^{x t}=\sum_{n=0}^{\infty} B_{n}^{(k)}(x, a, b, c) \frac{t^{n}}{n !}, \quad(1.10) \\
& |t|<\frac{2 \pi}{|\ln a+\ln b|}
\end{aligned}
$$

One can easily see that

and

$$
B_{n}^{(k)}(0,1, e)=B_{n}^{(k)}, B_{n}^{(k)}(x)=1+x
$$

$$
B_{n}^{(k)}(x)=B_{n}^{(k)}\left(e^{x+1}, e^{x}\right)
$$

where $B_{n}^{(k)}$ re generalized poly-Bernoulli numbers. For more information about poly-Bernoulli numbers and polyBernoulli polynomials, we refer to [15-20].

In [29-34] Pathan et al introduced the generalized Hermite-Bernoulli polynomials of two variables ${ }_{H} B_{n}^{(\alpha)}(x, y)$ is defined by

$$
\left(\frac{t}{e^{t}-1}\right)^{\alpha} e^{x t+y t^{2}}=\sum_{n=0}^{\infty} H_{n}^{(\alpha)}(x, y) \frac{t^{n}}{n !}
$$

which is essentially a generalization of Bernoulli numbers, Bernoulli polynomials, Hermite polynomials and HermiteBernoulli polynomials ${ }_{H} B_{n}(x, y)$ introduced by Dattoli et al [[14], p.386(1.6)] in the form

$$
\left(\frac{t}{e^{t}-1}\right) e^{x t+y t^{2}}=\sum_{n=0}^{\infty} H_{n}(x, y) \frac{t^{n}}{n !} .
$$

The Stirling number of the first kind is given by

$$
(x)_{n}=x(x-1) \cdots(x-n+1)=\sum_{l=0}^{n} S_{1}(n, l) x^{l},(n \geq 0)(1.14)
$$

and the Stirling number of the second kind is defined by generating function to be

$$
\left(e^{t}-1\right)^{n}=n ! \sum_{l=n}^{\infty} S_{2}(l, n) \frac{t^{l}}{l !}
$$

Recently many mathematicians have studied the symmetric identities on some special polynomials see for details [29-34,43,44]. Some of mathematicians also investigated some applications of poly-Bernoulli numbers and polynomials of the second kind cf. [26,27,28,36,37,38,39]. For more information about these polynomials, look at [1-44] and the references cited therein.

In this paper, we first give definitions of the Hermite poly-Bernoulli polynomials ${ }_{H} b_{n}^{(k)}(x, y)$ and we give some formulae of those polynomials related to the Stirling numbers of the second kind. Some implicit summation formulae and general symmetry identities are derived by using different analytical means and applying generating functions. These results extend some known summations and identities of generalized Hermite poly-Bernoulli numbers and polynomials of the second kind studied by Dattoli et al, Zhang et al, Yang, Khan, Pathan and Khan.

\section{Hermite poly-Bernoulli Numbers and Polynomials of the Second Kind}

For $k \in Z$, we consider the Hermite poly-Bernoulli polynomials ${ }_{H} b_{n}^{(k)}(x, y)$ of the second kind

$$
\frac{L i_{k}\left(1-e^{-t}\right)}{\log (1+t)}(1+t)^{x}\left(1+t^{2}\right)^{y}=\sum_{n=0}^{\infty}{ }_{H} b_{n}^{(k)}(x, y) \frac{t^{n}}{n !}(2.1)
$$

so that

$$
{ }_{H} b_{n}^{(k)}(x, y)=\sum_{m=0}^{n}\left(\begin{array}{l}
n \\
m
\end{array}\right) b_{n-m}^{(k)} H_{m}(x, y)
$$

when $x=y=0, b_{n}^{(k)}=b_{n}^{(k)}(0,0)$ are called the polyBernoulli numbers of the second kind. Indeed, for $k=1$ in (2.1), we have

$$
\frac{L i_{1}\left(1-e^{-t}\right)}{\log (1+t)}(1+t)^{x}\left(1+t^{2}\right)^{y}=\sum_{n=0}^{\infty}{ }_{H} b_{n}(x, y) \frac{t^{n}}{n !}
$$

Thus by (2.1) and (2.3), we get

$$
{ }_{H} b_{n}^{(k)}(x, y)={ }_{H} b_{n}(x, y),(n \geq 0) \text {. }
$$

For $y=0$ in (2.1), the result reduces to the polyBernoulli polynomials of the second kind Kim et al [[26], p.Eq.(7)2] is defined as

$$
\frac{L i_{k}\left(1-e^{-t}\right)}{\log (1+t)}(1+t)^{X}=\sum_{n=0}^{\infty} b_{n}^{(k)}(x) \frac{t^{n}}{n !},(k \in z) .
$$

Theorem 2.1. For $n \geq 0$, we have

$$
{ }_{H} b_{n}^{(2)}(x, y)=\sum_{m=0}^{n}\left(\begin{array}{l}
n \\
m
\end{array}\right) \frac{B_{m}}{m+1} H_{n-m}(x, y) .
$$

Proof. Applying Definition (2.1), we have 


$$
\begin{aligned}
& \sum_{n=0}^{\infty} H_{n} b_{n}^{(k)}(x, y) \frac{t^{n}}{n !}=\frac{L i_{1}\left(1-e^{-t}\right)}{\log (1+t)}(1+t)^{x}\left(1+t^{2}\right)^{y} \\
= & \frac{(1+t)^{x}\left(1+t^{2}\right)^{y}}{\log (1+t)} \\
& \int_{0}^{t} \frac{1}{e^{z}-1} \int_{0}^{t} \frac{1}{e^{z}-1} \cdots \frac{1}{e^{z}-1} \int_{0}^{t} \frac{z}{e^{z}-1} d z d z \cdots d z
\end{aligned}
$$

In particular $k=2$, we have

$$
\begin{aligned}
& \sum_{n=0}^{\infty} H_{n} b_{n}^{(2)}(x, y) \frac{t^{n}}{n !}=\frac{(1+t)^{x}\left(1+t^{2}\right)^{y}}{\log (1+t)} \int_{0}^{t} \frac{z}{e^{z}-1} d z \\
& =\left(\frac{t}{\log (1+t)}\right)(1+t)^{x}\left(1+t^{2}\right)^{y}\left(\sum_{m=0}^{\infty} \frac{t^{m} B_{m}}{(m+1) m !}\right) \\
& =\left(\sum_{n=0}^{\infty} H_{n} b_{n}(x, y) \frac{t^{n}}{n !}\right)\left(\sum_{m=0}^{\infty} \frac{t^{m} B_{m}}{(m+1) m !}\right) .
\end{aligned}
$$

Replacing $n$ by $n-m$ in above equation, we have

$$
=\sum_{n=0}^{\infty} \sum_{m=0}^{\infty}\left(\begin{array}{l}
n \\
m
\end{array}\right) \frac{B_{m}}{m+1}{ } b_{n-m}(x, y) \frac{t^{n}}{n !} .
$$

On equating the coefficients of the like powers of $t$ in the above equation, we get the result (2.5).

Remark 1. For $y=0$ in Theorem (2.1), the result reduces to known result of Kim et al [[26], p. 3, Theorem (2.1)].

Corollary 1. For $n \geq 0$, we have

$$
b_{n}^{(2)}(x)=\sum_{n=0}^{m}\left(\begin{array}{l}
n \\
m
\end{array}\right) \frac{B_{m}}{m+1} b_{n-m}(x) .
$$

Theorem 2.2. For $n \geq 0$, we have

$$
\begin{aligned}
& H_{n}^{(k)}(x, y) \\
& =\sum_{p=0}^{n}\left(\begin{array}{l}
n \\
p
\end{array}\right)\left(\sum_{l=1}^{p+1} \frac{(-1)^{l+p+1} l ! S_{2}(p+1, l)}{l^{k}(p+1)}\right){ }_{H} b_{n-p}(x, y)
\end{aligned}
$$

Proof. From equation (2.1), we have

$$
\begin{aligned}
& \sum_{n=0}^{\infty}{ }_{H} b_{n}^{(k)}(x, y) \frac{t^{n}}{n !}=\frac{L i_{k}\left(1-e^{-t}\right)}{\log (1+t)}(1+t)^{x}\left(1+t^{2}\right)^{y} \\
& =\frac{t}{\log (1+t)} \frac{L i_{k}\left(1-e^{-t}\right)}{t}(1+t)^{x}\left(1+t^{2}\right)^{y} .
\end{aligned}
$$

Now

$$
\begin{aligned}
& \frac{1}{t} L i_{k}\left(1-e^{-t}\right)=\frac{1}{t} \sum_{l=1}^{\infty} \frac{\left(1-e^{-t}\right)^{l}}{l^{k}}=\frac{1}{t} \sum_{l=1}^{\infty} \frac{(-1)^{l}}{l^{k}}\left(1-e^{-t}\right)^{l} \\
& =\frac{1}{t} \sum_{l=1}^{\infty} \frac{(-1)^{l}}{l^{k}} l ! \sum_{p=l}^{\infty}(-1)^{p} S_{2}(p, l) \frac{t^{p}}{p !} \\
& =\frac{1}{t} \sum_{p=1}^{\infty} \sum_{l=1}^{p} \frac{(-1)^{l+p}}{l^{k}} l ! S_{2}(p, l) \frac{t^{p}}{p !} \\
& =\sum_{p=0}^{\infty}\left(\sum_{l=1}^{p+1} \frac{(-1)^{l+p+1}}{l^{k}} l ! \frac{S_{2}(p+1, l)}{p+1}\right) \frac{t^{p}}{p !} .
\end{aligned}
$$

Thus by equations (2.3), (2.8) and (2.9), we get

$$
\begin{aligned}
& \sum_{n=0}^{\infty} H_{n} b_{n}^{(k)}(x, y) \frac{t^{n}}{n !} \\
& =\sum_{p=0}^{\infty}\left(\sum_{l=1}^{p+1} \frac{(-1)^{l+p+1}}{l^{k}} l ! \frac{S_{2}(p+1, l)}{p+1}\right) \frac{t^{p}}{p !} \sum_{n=0}^{\infty}{ }_{H} b_{n}(x, y) \frac{t^{n}}{n !} .
\end{aligned}
$$

Replacing $\mathrm{n}$ by $\mathrm{n}-\mathrm{p}$ in the r.h.s of above equation and comparing the coefficients of $t_{n}$, we get the result (2.7).

Remark 2. For $y=0$ in Theorem (2.2), the result reduces to known result of Kim et al [[26].,p. 4, Theorem (2.2)].

Corollary 2. For $n \geq 0$, we have

$$
\begin{aligned}
& b_{n}^{(k)}(x) \\
& =\sum_{p=0}^{n}\left(\begin{array}{l}
n \\
p
\end{array}\right)\left(\sum_{l=1}^{p+1} \frac{(-1)^{l+p+1} l ! S_{2}(p+1, l)}{l^{k}(p+1)}\right) b_{n=p}(x)
\end{aligned}
$$

Theorem 2.3. For $n \geq 1$, we have

$$
\begin{aligned}
& { }_{H} b_{n}^{(k)}(x+1, y)-{ }_{H} b_{n}^{(k)}(x, y) \\
& =\sum_{p=1}^{n} \sum_{l=1}^{p}\left(\begin{array}{l}
n \\
p
\end{array}\right) \frac{(-1)^{l+p}}{l^{k}} l ! S_{2}(p, l)_{H} b_{n-p}(x, y)
\end{aligned}
$$

Proof. Using the Definition (2.1), we have

$$
\begin{aligned}
& \sum_{n=0}^{\infty} H_{H} b_{n}^{(k)}(x+1, y) \frac{t^{n}}{n !}-\sum_{n=0}^{\infty} H_{n} b_{n}^{(k)}(x, y) \frac{t^{n}}{n !} \\
&= \frac{L i_{k}\left(1-e^{-t}\right)}{\log (1+t)}(1+t)^{x+1}\left(1+t^{2}\right)^{y} \\
&- \frac{L i_{k}\left(1-e^{-t}\right)}{\log (1+t)}(1+t)^{x}\left(1+t^{2}\right)^{y} \\
&= \frac{t L i_{k}\left(1-e^{-t}\right)}{\log (1+t)}(1+t)^{x}\left(1+t^{2}\right)^{y} \\
&=\left(\frac{t}{\log (1+t)}(1+t)^{x}\left(1+t^{2}\right)^{y}\right) \\
& \sum_{p=1}^{\infty}\left(\sum_{l=1}^{p} \frac{(-1)^{l+p}}{l^{k}} l ! S_{2}(p, l) \frac{t^{p}}{p !}\right. \\
&=\left(\sum_{n=0}^{\infty} H_{n} b_{n}(x, y) \frac{t^{n}}{n !}\right) \\
&\left(\sum_{p=1}^{\infty}\left(\sum_{l=1}^{p} \frac{(-1)^{l+p}}{l^{k}} l ! S_{2}(p, l) \frac{t^{p}}{p !}\right) .\right.
\end{aligned}
$$

Replacing $\mathrm{n}$ by $\mathrm{n}-\mathrm{p}$ in the above equation and comparing the coefficients of $t^{n}$, we get the result (2.11).

Remark 3. For $y=0$ in Theorem (2.3), the result reduces to known result of Kim et al [[26].,p. 4, Theorem (2.3)].

Corollary 3. For $n \geq 1$, we have

$$
\begin{aligned}
& b_{n}^{(k)}(x+1)-b_{n}^{(k)}(x) \\
& =\sum_{p=1}^{n} \sum_{l=1}^{p}\left(\begin{array}{l}
n \\
p
\end{array}\right) \frac{(-1)^{l+p}}{l^{k}} l ! S_{2}(p, l) b_{n-p}(x) .
\end{aligned}
$$


3. Implicit Summation Formulae Involving Hermite poly-Bernoulli Num-bers and Polynomials of the Second Kind

For the derivation of implicit formulae involving polyBernoulli polynomials of the second kind $b_{n}^{(k)}(x)$ and Hermite poly-Bernoulli polynomials of the second kind ${ }_{H} b_{n}^{(k)}(x, y)$ the same considerations as developed for the ordinary Hermite and related polynomials in Khan et al [21] and Hermite-Bernoulli polynomials in Pathan and Khan [29-34] holds as well. First we prove the following results involving Hermite poly-Bernoulli polynomials of the second kind ${ }_{H} b_{n}^{(k)}(x, y)$.

Theorem 3.1. For $x, y / R$ and $n \geq 0$. Then

$$
{ }_{H} b_{n}^{(k)}(x+u, y)=\sum_{j=0}^{n}\left(\begin{array}{c}
n \\
j
\end{array}\right)(-1)^{j}(-u)_{j H} b_{n-j}^{(k)}(x, y)(3.1)
$$

Proof. Since

$$
\begin{aligned}
& \sum_{n=0}^{\infty} H_{n} b_{n}^{(k)}(x+u, y) \frac{t^{n}}{n !} \\
& =\frac{\operatorname{Li}_{k}\left(1-(e)^{-t}\right)}{\log (1+t)}(1+t)^{(x+u)}\left(1+t^{2}\right)^{y} \\
& =\left(\sum_{n=0}^{\infty} H_{n}^{(k)}(x, y) \frac{t^{n}}{n !}\right)\left(\sum_{j=0}^{\infty}(-1)^{j}(-u)_{j} \frac{t^{j}}{j !}\right) .
\end{aligned}
$$

Now replacing $\mathrm{n}$ by $\mathrm{n}-\mathrm{j}$ and comparing the coefficients of $t^{n}$, we get the result (3.1).

Remark. Set $u=1$ in the above theorem to get

Corollary. For $x, y \in R$ and $n \geq 0$. Then

$$
{ }_{H} b_{n}^{(k)}(x+1, y)=\sum_{j=0}^{n}\left(\begin{array}{c}
n \\
j
\end{array}\right)(-1)^{j}{ }_{H} b_{n-j}^{(k)}(x, y) .
$$

Theorem 3.2. For $x, y / R$ and $n \geq 0$. Then

$$
\begin{aligned}
& { }_{H} b_{n}^{(k)}(x+u, y+w) \\
& =\sum_{m=0}^{n}\left(\begin{array}{l}
n \\
m
\end{array}\right) H b_{n-m}^{(k)}(x, y) H_{m}(u, w) .
\end{aligned}
$$

Proof. By the definition of poly-Bernoulli polynomials of the second kind and the definition (1.2), we have

$$
\begin{aligned}
& \frac{L i_{k}\left(1-(e)^{-t}\right)}{\log (1+t)}(1+t)^{(x+u)}\left(1+t^{2}\right)^{(y+w)} \\
& =\left(\sum_{n=0}^{\infty} H b_{n}^{(k)}(x, y) \frac{t^{n}}{n !}\right)\left(\sum_{m=0}^{\infty} H_{m}(u, w) \frac{t^{m}}{m !}\right) .
\end{aligned}
$$

Now replacing $\mathrm{n}$ by $\mathrm{n}-\mathrm{m}$ and comparing the coefficients of $t^{n}$, we get the result (3.3).

Theorem 3.3. For $x, y / R$ and $n \geq 0$. Then

$$
H^{b_{n}^{(k)}}(x, y)=\sum_{m=0}^{n-2 j} \sum_{j=0}^{\left[\frac{n}{2}\right]}\left[\begin{array}{l}
b_{m}^{(k)}(-y)_{j}(-1)^{n-m-j} \\
(-x)_{n-m-2 j} \frac{n !}{m ! j !(n-m-2 j) !}
\end{array}\right] .
$$

Proof. Applying the definition (2.1) to the term $\frac{L i_{k}\left(1-(e)^{-t}\right)}{\log (1+t)}$ and expanding the exponential function $(1+t)^{x}\left(1+t^{2}\right)^{y}$ at $t=0$ yields

$$
\begin{aligned}
& \frac{L_{k}\left(1-(e)^{-t}\right)}{\log (1+t)}(1+t)^{x}\left(1+t^{2}\right)^{y} \\
= & \left(\sum_{m=0}^{\infty} b_{m}^{(k)}(x, y) \frac{t^{m}}{m !}\right) \\
& \left(\sum_{n=0}^{\infty}(-x)_{n}(-1)^{n} \frac{t^{n}}{n !}\right) \\
& \left(\sum_{j=0}^{\infty}(-y)_{j}(-1)^{j} \frac{t^{2 j}}{j !}\right) \\
= & \sum_{n=0}^{\infty} \sum_{m=0}^{n-m}\left(\begin{array}{l}
n \\
m
\end{array}\right) b_{m}^{(k)}(-1)^{n-m}(-x)_{n-m} \frac{t^{n}}{n !} \sum_{j=0}^{\infty}(-y)_{j} \frac{t^{2 j}}{j !} .
\end{aligned}
$$

Replacing n by n-2j, we have

$$
\begin{aligned}
& \sum_{n=0}^{\infty} H^{b_{n}^{(k)}}(x, y) \frac{t^{n}}{n !} \\
& =\sum_{n=0}^{\infty} \sum_{m=0}^{n-2} \sum_{j=0}^{\left[\frac{n}{2}\right]}\left[\begin{array}{l}
b_{m}^{(k)}(-y)_{j}(-1)^{n-m-j} \\
\left.(-x)_{n-m-2 j} \frac{t^{n}}{m ! j !(n-m-2 j) !}\right] .
\end{array}\right.
\end{aligned}
$$

Equating their coefficients of $t^{n}$, we get the result (3.4).

Theorem 3.4. For $x, y \in R$ and $n \geq 0$. Then

$$
{ }_{H} b_{n}^{(k)}(x, y)=\sum_{r=0}^{n}\left(\begin{array}{l}
n \\
r
\end{array}\right)(-1)^{r}(-z)_{r H} b_{n-r}^{(k)}(x-z, y)
$$

Proof. Use the definition (2.1), we have

$$
\begin{aligned}
& \frac{L i_{k}\left(1-e^{-t}\right)}{\log (1+t)}(1+t)^{x-z}\left(1+t^{2}\right)^{y}(1+t)^{z} \\
& =\left(\sum_{n=0}^{\infty} H b_{n}^{(k)}(x-z, y) \frac{t^{n}}{n !}\right)\left(\sum_{r=0}^{\infty}(-1)^{r}(-z)_{r} \frac{t^{r}}{r !}\right) .
\end{aligned}
$$

Replacing $\mathrm{n}$ by $\mathrm{n}-\mathrm{r}$ in the above equation and comparing the coefficients of $t^{n}$, we get the result (3.6).

\section{General Symmetry Identity}

In this section, we give general symmetry identity for the poly-Bernoulli polynomials of the second kind $b_{n}^{(k)}(x)$ and the Hermite poly-Bernoulli polynomials of the second kind ${ }_{H} b_{n}^{(k)}(x, y)$ by applying the generating function(2.1) and (2.4). The results extend some known identities of Zhang and Yang [44], Yang [[43], Eqs.(9)], Khan [23,24,25] and Pathan et al [29-34].

Theorem 4.1. Let $a, b>0$ and $a \neq b$. For $x, y / R$ and $n \geq$ 0 . Then the following identity holds true: 


$$
\begin{aligned}
& \sum_{m=0}^{n}\left(\begin{array}{l}
n \\
m
\end{array}\right) b^{m} a^{n-m}{ }_{H} G_{n-m}^{(k)}\left(b x, b^{2} y\right)_{H} G_{m}^{(k)}\left(a x, a^{2} y\right) \\
& =\sum_{m=0}^{n}\left(\begin{array}{l}
n \\
m
\end{array}\right) a^{m} b^{n-m}{ }_{H} G_{n-m}^{(k)}\left(a x, a^{2} y\right)_{H} G_{m}^{(k)}\left(b x, b^{2} y\right)
\end{aligned}
$$

Proof. Start with

$$
\begin{aligned}
g(t)= & \left(\frac{\left(L i_{k}\left(1-e^{-t}\right)\right)^{2}}{(\log (1+a t))(\log (1+b t))}\right) \\
& \times(1+a b t)^{x}\left(1+a^{2} b^{2} t^{2}\right)^{y} .
\end{aligned}
$$

Then the expression for $\mathrm{g}(\mathrm{t})$ is symmetric in $\mathrm{a}$ and $\mathrm{b}$ and we can expand $g(t)$ into series in two ways to obtain

$$
\begin{aligned}
& g(t)=\frac{1}{a b} \sum_{n=0}^{\infty} H_{n}^{(k)}\left(b x, b^{2} y\right) \frac{(a t)^{n}}{n !} \\
& \times \sum_{m=0}^{\infty} H_{m}^{(k)}\left(a x, a^{2} y\right) \frac{(b t)^{m}}{m !} \\
& =\frac{1}{a b} \sum_{n=0}^{\infty} \sum_{m=0}^{n}\left(\begin{array}{l}
\left(\begin{array}{l}
n \\
m
\end{array}\right) a^{n-m_{H}} b_{H}^{m} b_{n-m}^{(k)}\left(b x, b^{2} y\right) \\
b_{m}^{(k)}\left(a x, a^{2} y\right) t^{n}
\end{array}\right)
\end{aligned}
$$

On the similar lines we can show that

$$
\begin{aligned}
& g(t)= \frac{1}{a b} \sum_{n=0}^{\infty} H_{n} b^{(k)}\left(a x, a^{2} y\right) \frac{(a t)^{n}}{n !} \\
& \times \sum_{m=0}^{\infty}{ }_{H}^{(k)}\left(b x, b^{2} y\right) \frac{(b t)^{m}}{m !} \\
&=\frac{1}{a b} \sum_{n=0}^{\infty} \sum_{m=0}^{n}\left(\begin{array}{l}
\left(\begin{array}{l}
n \\
m
\end{array}\right) a^{m} b^{n-m}{ }_{H} b_{n-m}^{(k)}\left(a x, a^{2} y\right) \\
{ }_{H}^{(k)}\left(b x, b^{2} y\right) t^{n}
\end{array}\right)
\end{aligned}
$$

Comparing the coefficients of $t^{n}$ on the right hand sides of the last two equations we arrive the desired result.

Remark 1. By setting $b=1$ in Theorem 4.1, we immediately following result

\section{Corollary.}

$$
\begin{aligned}
& \sum_{m=0}^{n}\left(\begin{array}{l}
n \\
m
\end{array}\right) a^{n-m} b_{H}^{m} b_{n-m}^{(k)}(x, y)_{H} b_{m}^{(k)}\left(a x, a^{2} y\right) \\
& =\sum_{m=0}^{n}\left(\begin{array}{l}
n \\
m
\end{array}\right) a_{H}^{m} b_{n-m}^{(k)}\left(a x, a^{2} y\right)_{H} b_{m}^{(k)}(x, y) .
\end{aligned}
$$

\section{Conclusion}

Based on the definition of Hermite polynomials and poly logarithmic function, we introduced a new class of Hermite poly-Bernoulli numbers and polynomials of the second kind. By using Jolany's methods introduced in [17] and [18], we gave Hermite poly-Bernoulli numbers and polynomials of the second kind with two variable, and also we analyse its behaviors including general symmetric properties.

\section{References}

[1] Andrews, L.C: Special functions for engineers and mathematicians, Macmillan. Co.New York, 1985

[2] Apostol, T.M: On the Lerch zeta function, Pacific J.Math. 1(1951), 161-167.

[3] Araci, S: Novel identities for q-Genocchi numbers and polynomials, J. Funct.Spaces Appl. 2012 (2012) 13p (Article ID 214961).

[4] Araci, S: Novel identities involving Genocchi numbers and polynomials arisingfrom applications of umbral calculus, Appl.Math. and Comput. 233(2014), 599-607.

[5] Araci, S, M. Acikgoz, M, Seo, J.J: Explicit formulas involving qEuler numbers and polynomials, Abstr. Appl. Anal. 2012 (2012) 11p (Article ID 298531).

[6] Araci, S, Erdal, D and Seo, J.J: A study on the fermionic p-adic qintegral representation on Zp associated with weighted q-Bernstein and q-Genocchi polynomials, Abstr. Appl. Anal. 2011 (2011) 10p (Article ID 649248).

[7] Araci, S, Acikgoz, M, Jolany, H, Seo, J.J: A unified generating function of the q-Genocchi polynomials with their interpolation functions, Proc. Jangjeon Math. Soc. 15 (2) (2012), 227-233.

[8] Araci, Sen, E, Acikgoz, M: A note on the modified q-Dedekind sums, Notes Number Theory Discrete Math. 19 (3) (2013), 60-65.

[9] Araci, A, Acikgoz, S M, Bagdasaryan, A, Sen, E: The Legendre polynomials associated with Bernoulli, Euler, Hermite and Bernstein polynomials, Turkish J.Anal. Number Theory (1) (2013) 13.

[10] Acikgoz, M, Araci, S, Cangul, I.N: A note on the modified qBernstein polynomials for functions of several variables and their reflections on q-Volkenborn integration, Appl. Math. Comput. 218 (3) (2011), 707-712.

[11] Araci, Sen, E, Acikgoz, M: Theorems on Genocchi polynomials of higher order arising from Genocchi basis, Taiwanese. J.Math. 18(2014), 473-482.

[12] Araci, Acikgoz, M, Sen, E: On the von Staudt-Clausen's theorem associated with q-Genocchi numbers, Appl.Math. and Comput. 247(2014), 780-785.

[13] Bell, E.T: Exponential polynomials, Ann. of Math. 35(1934), 258277.

[14] Dattoli, G, Lorenzutta, S and Cesarano, C: Finite sums and generalized forms of Bernoulli polynomials Rendiconti di Mathematica, 19(1999), 385-391.

[15] Hamahata, Y, Masubuch, H: Special Multi-Poly- Bernoulli numbers, Journal of Integer sequences,10 (2007), 1-6.

[16] Hamahata, Y, Masubuchi, H: Recurrence formulae for Multi-polyBernoulli numbers, Elec. J.Comb.Num.Theo. 7(2007), A-46.

[17] Jolany, H, Darafsheh, M.R, Alikelaye,R.E: Generalizations of Poly-Bernoulli Numbers and Polynomials, Int. J. Math. Comb. 2 (2010), A07-14.

[18] Jolany, H, Corcino, R.B: Explicit formula for generalization of Poly-Bernoulli numbers and polynomials with a,b,c parameters, Journal of Classical Analysis, 6(2015), 119-135.

[19] Jolany, H, Aliabadi, M, Corcino, R. B and Darafsheh, M.R: A Note on Multi Poly-Euler Numbers and Bernoulli Polynomials, General Mathematics, 20(2-3) (2012), 122-134.

[20] Jolany, $\mathrm{H}$ and Corcino, R.B: More properties on Multi-Euler polynomials, arXiv;1401.627IvI[math NT] 24 Jan 2014.

[21] Khan, S, Pathan, M.A, Hassan, Nader Ali Makhboul , Yasmin, G: Implicit summation formula for Hermite and related polynomials, J.Math.Anal.Appl. 344(2008), 408-416.

[22] Kaneko, M: Poly-Bernoulli numbers, J.de Theorie de Nombres 9 (1997), 221-228.

[23] Khan W.A: Some properties of the generalized Apostol type Hermite-Based polynomials, Kyungpook Math. J., 55(2015), 597614.

[24] Khan W.A, A note on Hermite-based poly-Euler and multi polyEuler polynomials, Palestine J. Math. vol 5(1) (2016), 17-26.

[25] Khan W.A: A new class of Hermite poly-Genocchi polynomials, J.Anal. and Number. Theory, 4(1) (2016), 1-8. 
[26] Kim, T, Kwaon, H.I, Lee, S.H and Seo, J.J: A note on polyBernoulli numbers and polynomials of the second kind, Advances in Defference Equations, (2014), 2014:219.

[27] Kim, D.S, Kim, T Mansour, T and Dolgy, D.V: On poly-Bernoulli polynomials of this second kind with umbral calculus view point, Advances Diffrences Equations, (2015), 2015:27.

[28] Kim, T, Kwon, H.I, Seo, J.J: On $\lambda$-Bernoulli polynomials of the second kind, Appl.Math.Scien., vol.9 (2015), 5275-5281.

[29] Pathan, M.A and Khan, W.A: Some implicit summation formulas and symmetric identities for the generalized Hermite basedpolynomials, Acta Universitatis Apulensis, 39(2014), 113-136.

[30] Pathan, M.A and Khan, W.A: Some implicit summation formulas and symmetric identities for the generalized Hermite-Bernoulli polynomials, Mediterr. J. Math. 12 (2015), 679-695.

[31] Pathan, M.A and Khan, W.A: A new class of generalized polynomials associated with Hermite and Euler polynomials , To appear in Mediterr. J. Math. Springer Basel 2015.

[32] Pathan, M.A and Khan, W.A:Some implicit summation formulas and symmetric identities for the generalized Hermite-Euler polynomials, East-West J.Maths. 16(1) (2014), 92-109.

[33] Pathan, M.A and Khan, W.A: A new class of generalized polynomials associated with Hermite and Bernoulli polynomials, LE MATEMATICHE, Vol. LXX (2015), 53-70

[34] Pathan, M.A and Khan, W.A: Some new classes of generalized Hermite-based Apostol-Euler and Apostol-Genocchi polynomials, Fasciculli.Math. Vol.55(2015), In Press.
[35] Park,J.W and Rim, S.H: On the modified q-Bernoulli polynomials with weight, Proc.Jangjeon Math. Soc., 17 (2014), no. 2, 231-236.

[36] Qi, F and Guo, B.N: Explicit formulas for special values of the Bell polynomials of the second kind and for the Euler numbers and polynomials, September 2014.

[37] Qi, F: Explicit formulas for computing Bernoulli numbers of the second kind and stirling numbers of the first kind, Filomat, 28:2(2014), 319-327.

[38] Qi, F: A new formula for the Bernoulli numbers of the second kind in terms of the stirling numbers of the first kind, Publications De L'Institute Mathematique.

[39] Qi, F. Kim, D.S, Kim, T and Dolgy, D.V: Multiple poly-Bernoulli polynomials of the second kind, Advanced Studies in Contemporary Mathematics, 25(2015), 1-7.

[40] Sandor, J and Crisci: Handbook of Number Theory, Vol.II. Kluwer Academic Publishers, Dordrecht Boston and London, 2004.

[41] Srivastava, H.M and Choi, J: Series associated with the Zeta and related functions, Kluwer Academic Publishers, Dordrecht, Boston and London, 2001.

[42] Srivastava, H.M and Pinter, A: Remarks on some relationships between the Bernoulli and Euler polynomials, Appl.Math.Lett. 17(2004), 375-380.

[43] Yang, S: An identity of symmetry for the Bernoulli polynomials, Discrete Math. Vol. 308 (2008), 550-554.

[44] Zhang, Z and Yang, H: Several identities for the generalized Apostol-Bernoulli polynomials, Computers and Mathematics with Applications, 56(2008), 2993-2999. 